\title{
Advances in Reconstruction of the Skull Base
}

\author{
Rounak B. Rawal $\cdot$ Emily C. Ambrose $\cdot$ \\ Mihir R. Patel · Adam M. Zanation
}

Published online: 8 September 2013

(c) Springer Science+Business Media New York 2013

\begin{abstract}
Reconstruction of the skull base continues to advance at an exponentially rapid rate. New methods of vascular tissue mobilization, improvement in technology, and advancement in minimally invasive tissue harvesting allow for multiple endoscopic reconstruction options of the anterior skull base. Size and location of defect, along with experience of surgical technique, all play a part in the algorithm for choice of reconstructive method. Reduction of nasal morbidity has increased, while rates of complications continue to decline with increasing surgical experience and technique. Continued efforts to expand the armamentarium of the skull base surgeon will advance the field towards better clinical outcomes and reduced complications. Here, we review the most current avascular and vascular reconstructive options available, highlight recent advances in decreasing patient morbidity, and discuss complications occurring during reconstruction.
\end{abstract}

Keywords Skull base $\cdot$ Reconstruction - Nasoseptal flap · Vascular flap

\section{Introduction}

Since the popularization of the endoscopic endonasal approach (EEA) for intradural pathology, EEA has allowed

R. B. Rawal · E. C. Ambrose - A. M. Zanation $(\bowtie)$

Department of Otolaryngology - Head and Neck Surgery, University of North Carolina-Chapel Hill, 170 Manning Dr., Physician Office Building, Ground Floor, CB\#7070, Chapel Hill, NC 27599, USA

e-mail: adam_zanation@med.unc.edu

M. R. Patel

Department of Otolaryngology - Head and Neck Surgery, University of Pennsylvania, HUP - 5th Floor Ravdin, 34th and Spruce Streets, 4283, Philadelphia, PA 19104, USA surgeons access to the entire ventral skull base, including the anterior, middle, and posterior cranial fossae (see Fig. 1 for case example) [1,2]. Reconstruction of defects created during the EEA presents a unique challenge, as factors such as size of defect, availability of graft and vascular flaps, technical difficulty, and morbidity of the patient all impact the decision-making process. In general, smaller defects $(<1 \mathrm{~cm})$ may be repaired with avascular grafts while larger $(>3 \mathrm{~cm})$ defects require vascularized flaps for reliable reconstruction (Fig. 1) [3].

Goals of reconstruction of the anterior skull base, whether open or endoscopic, remain the same. A watertight seal is striven for, in order to prevent cerebrospinal fluid (CSF) leakage from the cranial vault, to prevent ascending infection from the pharynx, and to prevent the possibility of future pneumocephalus. A reconstructive plan must always be specifically tailored for the individual patient, as dural defects are rarely exactly alike. Certain clinical scenarios may preclude use of a particular reconstructive method and warrant one that is less often used. It is therefore imperative that the skull base surgeon be comfortable with a variety of reconstructive methods. Advances in skull base reconstruction over the last year have focused on expanding secondary options for revision patients, reducing postoperative morbidity during the healing process and increasing the evidence for outcomes and utilization of vascular flaps via systematic reviews.

\section{Avascular Grafts}

A variety of materials and methods exist for reconstruction of defects smaller than $1 \mathrm{~cm}$. Avascular grafts include autologous grafts, acellular human dermis grafts (Alloderm; LifeCell, Branchburg, NJ, USA), and engineered collagen grafts. 
Fig. 1 Sagittal (a) and coronal (b) views of 2-year postoperative MRI of endoscopic intradural craniofacial reconstruction showing contrast enhancing flap from the planum to the frontal sinus with $100 \%$ skull base and dural defect coverage
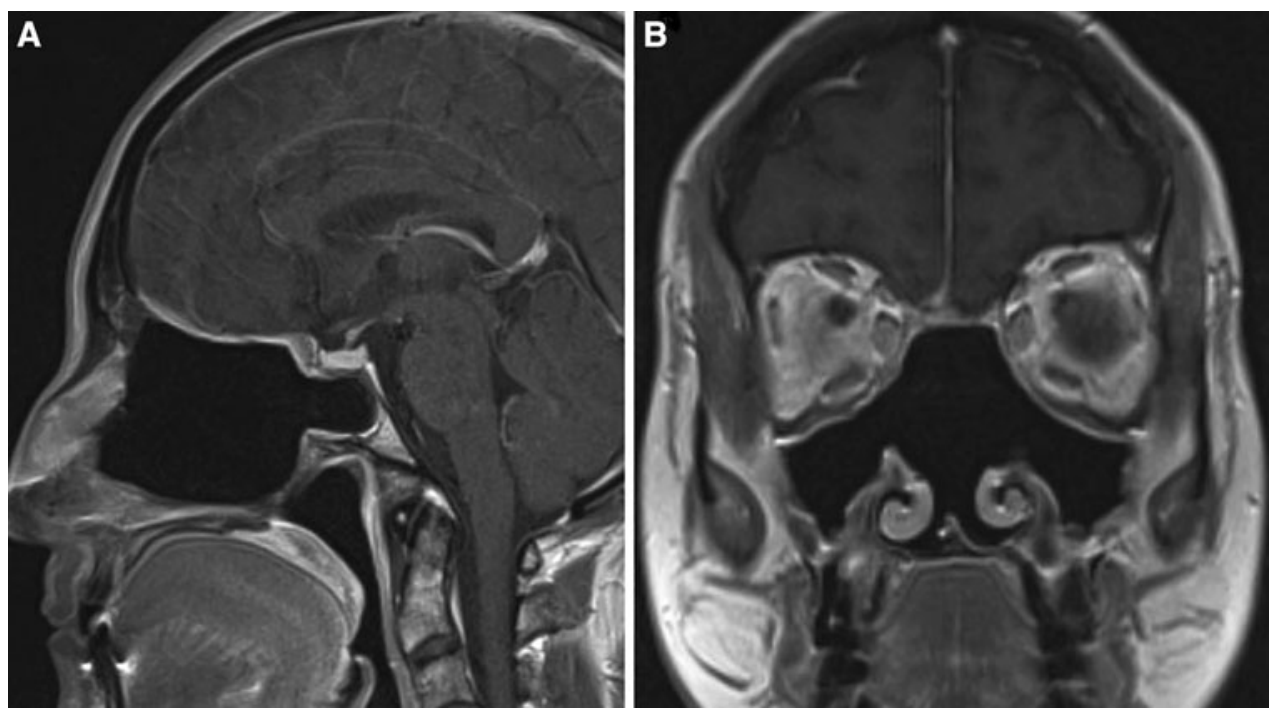

Autologous grafts include fat, fascia, bone, cartilage, and free mucosa grafts harvested from the patient [4]. Advantages of autologous grafts include negligible cost, near-universal availability, and biocompatibility between graft and host. Disadvantages include a second surgical incision for the patient, additional healing time, increased operating room time for additional tissue harvest, and additional discomfort for the patient [5]. Acellular human dermis grafts have the advantage of requiring no additional surgical incision, being relatively cheap, and being relatively easy to use, but there are reports of atrophy or decreased adhesion with the brain surface [6]. Engineered collagen grafts (i.e., DuraGen; Integra Neurosciences, Plainsboro, NJ, USA), thought to serve as a scaffolding for indigenous cell growth, can be applied in a sutureless method, but may predispose the patient to postoperative complications and increase the cost of surgery [7].

The current literature cannot objectively evaluate the success of one avascular graft over the other, as most data series are retrospective and do not control for adjuvant reconstructive materials nor for the method of reconstruction (overlay vs. underlay vs. multilayered reconstruction, etc.) [4]. In addition, most studies solely use recurrence of CSF leak as the primary and only endpoint for surgical success [8]. While important, this endpoint has shown that, in experienced hands, choice of reconstruction does not seem to matter, as most grafts have a $90 \%$ initial success rate of CSF leak repair with a $97 \%$ success rate upon secondary repair regardless of graft used [9]. Further studies using other endpoints may shed further light in providing a definitive answer for first-line reconstructive material to be used for a $<1 \mathrm{~cm}$ small dural defect.

For small skull base defects, data do not support a difference in outcomes by reconstructive techniques or grafting materials. This is in stark contrast to large dural defects. A recently published systematic review presented a $15.6 \%$ leak rate for free grafts and a $6.7 \%$ leak rate for the vascularized reconstructions $(p=.001)$, significantly supporting the use of vascular reconstructions for large dural defects $\left[10^{\bullet}\right]$. Due to the increased utilization and success of vascular flaps for endoscopic skull base reconstruction, several intranasal and local options have been developed (Table 1).

\section{Nasoseptal Flap}

Since its introduction by Hadad and Bassagaisteguy, the vascular pedicle nasoseptal flap (NSF) has become the vascular flap of choice for skull base reconstruction [11]. The NSF is comprised of harvested mucoperiosteum and mucopericondrium pedicled on the nasoseptal artery, a branch of the posterior septal artery, itself a terminal branch of the internal maxillary artery [11]. Techniques of the NSF have been described elsewhere and are beyond the scope of this review article. [3, 11, 12].

The NSF provides several distinct advantages. Radioanatomic studies and clinical case series have proven that the NSF can cover anterior cranial fossa, transsphenoidal, and transclival defects independently for patients greater than 14 years of age, with an average coverage of $25 \mathrm{~cm}^{2}$ of surface area. [12-14] The NSF enjoys a high degree of success after skull base reconstruction (95\% and greater), and has reliably been used in multiple institutions and countries $[15,16]$. Modifications of the NSF have provided a myriad of reconstructive options with high success rates, from takedown and reuse of the NSF during revision surgery years after the primary surgery (95\%) [17], to creating a bilateral "Janus flap" for an extremely large dural defect $(100 \%)$ [18], to partial harvest of the NSF as a nasoseptal "rescue" flap (NSRF) to be used only in case of CSF leak (100\%) [19•]. 
Table 1 Vascular flaps for skull base reconstruction
$A C F$ anterior cranial fossa, $M C F$ middle cranial fossa, $F T T$ free tissue transfer

\begin{tabular}{llll}
\hline Location & Flap & Pedicle & Defects reconstructed \\
\hline Intranasal & Anterior lateral nasal wall & Angular artery & ACF \\
& Anferior turbinate & Inferior turbinate artery & Sella \\
& & & Clivus \\
& Middle turbinate & Middle turbinate artery & ACF \\
& & & Transsphenoidal \\
Regional & Nasoseptal flap & Sphenopalatine artery & All \\
& Endoscopic-assisted pericranial & Supraorbital artery & ACF to Sella \\
& & Supratrochlear artery & \\
& Facial artery buccinator & Angular artery & ACF \\
& Occipital & & Parasellar \\
& & Occipital artery & Clivus \\
& Palatal & & Coronal plane of the MCF \\
& & Greater palatine artery & Sella \\
& & & Planum \\
& Tunneled temporoparietal fascia & Superficial temporal artery & Parasellar \\
& & & Clivus \\
& Various & Various & All \\
\hline
\end{tabular}

A disadvantage of the NSF may include its surgical complexity, but it is a complexity whose struggle bears fruit, as authors have noted significant reduction in postoperative CSF leaks with increasing numbers of cases [20]. A theoretical disadvantage of the NSF is the greater risk of insetting the flap over intact underlying mucosa and subsequent future mucocele formation, but long-term studies have not show this to be the case. [21].

\section{Advancements in Secondary Vascularized Flaps}

While the NSF is the primary workhorse of skull base reconstruction, there are times in which the NSF may be unavailable for utilization. This may occur when prior radiation or surgery has extensively damaged the vascular supply to the NSF, when a previous posterior septectomy has been done, or when there is direct tumor invasion of the nasal septum [22]. During these situations, we look to use other vascularized skull base reconstruction options.

\section{Intranasal Flaps}

When the NSF is unavailable, the second-line choice for reconstruction is any of the other locoregional intranasal flaps that may be available. There are three other intranasal options: the anterior lateral nasal wall flap (ALNWF), the interior turbinate flap (ITF), and the middle turbinate flap (MTF). In general, the ALNWF is the most versatile of the intranasal flaps. The ALNWF is pedicled on the branches of the facial (angular and lateral nasal) and anterior ethmoidal arteries and incorporates the mucosa from the nasal lateral wall and floor [23]. A major advantage of the ALNWF is the ability to combine the ALNWF with a NSF for repair of very large dural defects.

The ITF is a flap pedicled on the interior turbinate artery, a terminal branch of the posterior lateral nasal artery, itself a branch from the sphenopalatine artery [24]. Cadaveric studies have shown that the ITF may cover the anterior skull base from the posterior table of the frontal sinus to the sella, with an average of $4.97 \mathrm{~cm}^{2}$ surface area coverage $[14,25]$. The major disadvantages of the ITF are the decrease in surface area that the ITF can provide for skull base repair and the decrease in length for flap placement as compared to other locoregional vascularized flaps [24, 26, 27].

The MTF is a flap pedicled on branches of the sphenopalatine artery, along with branches of the anterior septal arteries [14]. The MTF is adequate to provide coverage for transplanar, transtubercular, and/or transsellar defects, and can provide an average $5.6 \mathrm{~cm}^{2}$ of surface area coverage [14]. The disadvantage of the MTF lies in its smaller surface area coverage, the highly variable pneumatization of the middle turbinate, and the presence of bullous turbinates [28].

\section{Tunneled Scalp Flaps}

The endoscopically-harvested pericranial flap (PCF) is one of the most commonly used second-line vascular flaps. 
This is mainly because the same reasons that would exclude the use of a NSF (i.e., sinonasal tumorigenic invasion, previous septectomy, etc.) usually also rule out other intranasal pedicled flaps. As such, we then turn to tunneled scalp flaps, including the PCF and the temporoparietal fascia flap (TPFF). The PCF's strong vascularity relies on its pedicle on the deep branches of the supraorbital and the supratrochlear arteries, and it can extend from the superior orbital rim down to the occiput [29, 30]. Techniques of the endoscopically-harvested PCF have been described elsewhere and are beyond the scope of this review article [31, 32]. Advantages of the PCF include a less technically demanding surgical procedure, a greater length and surface area coverage of the flap (up to $22 \mathrm{~cm}^{2}$ ), and a greater radioresistance, allowing for reconstruction in sites that will require postoperative radiation [31, 33]. In addition, favorable cosmesis can be achieved with only a $1-\mathrm{cm}$ short incision made in a glabellar skin crease. Disadvantages of the PCF include difficulty with tissue harvest, especially when a patient's forehead is short, his or her hairline is low, or his or her forehead is particularly protuberant [31].

The tunneled TPFF is pedicled on branches from the superficial temporal artery, a terminal branch of the external carotid artery [34]. The TPFF allows for extremely large dural defect coverage of up to $17 \times 14 \mathrm{~cm}$, making it one of the most versatile locoregional pedicled flaps [35]. While open approaches to the TPFF have been described in many articles, an endoscopic approach requires a hemicoronal incision for harvest of the flap, along with tunneling through the ipsilateral temporal-infratemporal region into the nasal cavity via an endonasal transpterygoid approach [34]. Although it has successfully been used in the postradiated patient, care must be taken due to the possibility of extensive scalp exposure to radiation and subsequent ischemia at the donor site upon TPFF harvest [34]. Other disadvantages include the technically challenging nature of the procedure, as we believe the TPFF requires the highest surgical experience for successful use amongst all locoregional vascular pedicled flaps.

\section{Other Regional Flaps}

Due to the myriad ways in which dural defects can present, it is important for the skull base surgeon to have knowledge and comfort with other secondary and tertiary flap options for reconstruction. While these flaps may provide for unique dural injury, they come with their own possible morbidities due to their unique anatomic locations.

The facial artery buccinator flap (FABF) is pedicled on the angular artery, a branch of the facial artery [36]. In contrast to the NSF and the ITF, the FABF can be harvested and transposed following the surgical resection [36].
Disadvantages include possible patient dental and facial paresthesias, persistent epiphora, and the theoretical direct spread of bacterial flora from the oral cavity to dural defects.

The modified palatal flap (PF), pedicled on the descending palatine artery, allows for transposition by enlargement of the greater palatine foramen [37]. The PF can cover a surface area of up to $18 \mathrm{~cm}^{2}$, and is adequate to cover planum, sellar, and clival defects down to the level of the foramen magnum because of its 3 -cm-long pedicle [37]. The greatest danger with the modified PF is the possibility for oroantral fistula formation, although combining the PF with an acellular dermis graft such as Alloderm may avert this [38].

The occipital flap (OF) is pedicled on the occipital artery, a consistent and highly predictable vascular supply, and has been described in the literature extensively. The OF can cover a surface area of up to $14 \times 4 \mathrm{~cm} \mathrm{[39].} \mathrm{The}$ novel transparapharyngeal-transpterygoid transposition allows for placement of the OF through the nasopharynx to repair anterior and middle cranial fossa defects, especially those found in the coronal plane across the skull base floor [40].

\section{Advances in Patient Morbidity Reduction}

Commonly cited disadvantages for the use of vascularized flaps are the additional incision, operating time, and patient morbidity required for tissue harvest. However, three recent advances have sought to minimize these perceived disadvantages of vascularized flaps.

One method of patient morbidity reduction has been through the introduction of the NSRF [41]. One of the pitfalls of using the NSF during EEA is that the NSF must be raised prior to beginning surgery. Thus, NSFs would be raised needlessly for patients in whom dural defects are not encountered. To counter this, the NSRF modifies the NSF by harvesting the most superior and posterior aspect of the flap, thereby providing pedicle protection and access to the sphenoid face during the approach concomitantly. The rescue flap can then be fully harvested at the end of the case if the resultant defect is larger than expected or if an unexpected CSF leak develops. If no such event occurs, the rescue flap can be reapproximated over the septal posterior septectomy defect, making sure that the sphenoid os can drain in its natural position. Initial case series have shown high degrees of success, with up to $98 \%$ of patients saving unnecessary NSRF harvest during EEA [19•, 42•, 43•]. Additionally, this reconstructive method has been found to improve exposure intra-operatively, preserve septal mucosa and the sphenopalatine artery, and reduce the rate of post-operative nasal complications [19•, 42•, 43•]. 
A second technique to reduce patient morbidity during the NSF is graft placement of free middle turbinate mucosal grafts onto the denuded septum during EEA [44•, 45]. Instead of simply discarding the resected middle turbinate, the middle turbinate is denuded of its mucosa and mucoperiosteum, and this graft is secured to the area of exposed cartilage on the nasal septum where the NSF had been harvested. Use of native nasal mucosa has been associated with increased rates of mucosalization. As early as 3 weeks post-operatively, there is a substantially higher rate of septal remucosalization in patients with middle turbinate grafts with almost complete mucosalization at 6 weeks [43•]. Additionally, free turbinate mucosal grafts have been associated with decreased rates of crusting and virtual no increase in cost [44•].

A third alternative to decrease patient morbidity at the donor site is the reverse rotation flap. The reverse rotation flap involves creation of a contralateral anteriorly-based nasal mucosal flap to redrape denuded septum from a NSF harvest $[46,47 \cdot]$. This technique has also improved postoperative quality of life by quickening septal remucosalization, decreasing nasal crusting formation, requiring fewer nasal irrigations, and creating a better nasal airway after use of the NSF [46]. Given these benefits, this flap is a reliable and successful adjunct to any NSF.

\section{Conclusion}

Reconstruction of the anterior skull base requires ingenuity, creativity, and a team-based approach comprised of otolaryngologists, neurosurgeons, plastic surgeons, ophthalmologists, and oral and maxillofacial surgeons. Although we have attempted to individually parse out the differences amongst grafts, flaps, and techniques to decrease patient morbidity, reality may require the use of multiple techniques and reconstruction methods for reconstruction. Reconstruction methods and options are constantly evolving, and continued familiarity with the most recent advances in skull base reconstruction is a necessity for the skull base surgeon [48, 49].

\section{Compliance with Ethics Guidelines}

Conflict of Interest Rounak B. Rawal, Emily C. Ambrose, Mihir R. Patel, and Adam M. Zanation declare that they have no conflict of interest.

Human and Animal Rights and Informed Consent This article does not contain any studies with human or animal subjects performed by any of the authors.

\section{References}

Papers of particular interest, published recently, have been highlighted as:
- Of importance

- Of major importance

1. Jho HD, Carrau RL. Endoscopic endonasal transsphenoidal surgery: experience with 50 patients. J Neurosurg. 1997;87(1): 44-51.

2. Snyderman CH, Pant H, Carrau RL, et al. What are the limits of endoscopic sinus surgery?: the expanded endonasal approach to the skull base. Keio J Med. 2009;58(3):152-60.

3. Zanation AM, Thorp BD, Parmar P, et al. Reconstructive options for endoscopic skull base surgery. Otolaryngol Clin North Am. 2011;44(5):1201-22.

4. Prickett KK, Wise SK. Grafting materials in skull base reconstruction. Adv Otorhinolaryngol. 2013;74:24-32.

5. Burkett CJ, Patel S, Tabor MH, et al. Polyethylene glycol (PEG) hydrogel dural sealant and collagen dural graft matrix in transsphenoidal pituitary surgery for prevention of postoperative cerebrospinal fluid leaks. J Clin Neurosci. 2011;18(11):1513-7.

6. Parlato $C$, di Nuzzo G, Luongo $M$, et al. Use of a collagen biomatrix (TissuDura) for dura repair: a long-term neuroradiological and neuropathological evaluation. Acta Neurochir (Wien). 2011;153(1):142-7.

7. Stendel R, Danne M, Fiss I, et al. Efficacy and safety of a collagen matrix for cranial and spinal dural reconstruction using different fixation techniques. J Neurosurg. 2008;109(2):215-21.

8. Prickett KK, Wise SK, Delgaudio JM. Choice of graft material and postoperative healing in endoscopic repair of cerebrospinal fluid leak. Arch Otolaryngol Head Neck Surg. 2011;137(5): 457-61.

9. Hegazy HM, Carrau RL, Snyderman CH, et al. Transnasal endoscopic repair of cerebrospinal fluid rhinorrhea: a metaanalysis. Laryngoscope. 2000;110(7):1166-72.

10. • Harvey RJ, Parmar P, Sacks R, et al., Endoscopic skull base reconstruction of large dural defects: a systematic review of published evidence. Laryngoscope. 2012; 122(2): 452-9. This is a systematic review and meta-analysis compiling all known case series, retrospective cohorts, and case-control studies comparing different methods of reconstruction for large $(>3 \mathrm{~cm})$ dural defects. Vascularized reconstructions had a significantly reduced rate of CSF leaks as compared to free grafts $(p=0.001)$.

11. Hadad G, Bassagasteguy L, Carrau RL, et al. A novel reconstructive technique after endoscopic expanded endonasal approaches: vascular pedicle nasoseptal flap. Laryngoscope. 2006;116(10):1882-6.

12. Pinheiro-Neto CD, Prevedello DM, Carrau RL, et al. Improving the design of the pedicled nasoseptal flap for skull base reconstruction: a radioanatomic study. Laryngoscope. 2007;117(9): 1560-9.

13. Shah RN, Surowitz JB, Patel MR, et al. Endoscopic pedicled nasoseptal flap reconstruction for pediatric skull base defects. Laryngoscope. 2009;119(6):1067-75.

14. Prevedello DM, Barges-Coll J, Fernandez-Miranda JC, et al. Middle turbinate flap for skull base reconstruction: cadaveric feasibility study. Laryngoscope. 2009;119(11):2094-8.

15. Thakur B, Jesurasa AR, Ross R, et al. Transnasal trans-sphenoidal endoscopic repair of CSF leak secondary to invasive pituitary tumours using a nasoseptal flap. Pituitary. 2011;14(2): $163-7$.

16. El-Sayed IH, Roediger FC, Goldberg AN, et al. Endoscopic reconstruction of skull base defects with the nasal septal flap. Skull Base. 2008;18(6):385-94.

17. Zanation AM, Carrau RL, Snyderman $\mathrm{CH}$, et al. Nasoseptal flap takedown and reuse in revision endoscopic skull base reconstruction. Laryngoscope. 2011;121(1):42-6. 
18. Nyquist GG, Anand VK, Singh A, et al. Janus flap: bilateral nasoseptal flaps for anterior skull base reconstruction. Otolaryngol Head Neck Surg. 2010;142(3):327-31.

19. -Rawal RB, Kimple AJ, Dugar DR, et al., Minimizing morbidity in endoscopic pituitary surgery: outcomes of the novel nasoseptal rescue flap technique. Otolaryngol Head Neck Surg. 2012; 147(3): 434-7. This paper describes the technique of the nasoseptal rescue flap (NSRF) and retrospective outcomes of the first cohort of patients with the NSRF with a $100 \%$ CSF leak closure rate.

20. Kassam AB, Thomas A, Carrau RL, et al. Endoscopic reconstruction of the cranial base using a pedicled nasoseptal flap. Neurosurgery. 2008;63(1 Suppl 1):ONS44-52 discussion ONS52-3.

21. Bleier BS, Wang EW, Vandergrift WA 3rd, et al. Mucocele rate after endoscopic skull base reconstruction using vascularized pedicled flaps. Am J Rhinol Allergy. 2011;25(3):186-7.

22. Zanation AM, Carrau RL, Snyderman CH, et al. Nasoseptal flap reconstruction of high flow intraoperative cerebral spinal fluid leaks during endoscopic skull base surgery. Am J Rhinol Allergy. 2009;23(5):518-21.

23. Hadad G, Rivera-Serrano CM, Bassagaisteguy LH, et al. Anterior pedicle lateral nasal wall flap: a novel technique for the reconstruction of anterior skull base defects. Laryngoscope. 2011;121(8):1606-10.

24. Fortes FS, Carrau RL, Snyderman CH, et al. The posterior pedicle inferior turbinate flap: a new vascularized flap for skull base reconstruction. Laryngoscope. 2007;117(8):1329-32.

25. Amit M, Cohen J, Koren I, et al. Cadaveric study for skull base reconstruction using anteriorly based inferior turbinate flap. Laryngoscope. 2013. doi:10.1002/lary.24186.

26. Yip J, Macdonald KI, Lee J, et al. The inferior turbinate flap in skull base reconstruction. J Otolaryngol Head Neck Surg. 2013;42(1):6.

27. Harvey RJ, Sheahan PO, Schlosser RJ. Inferior turbinate pedicle flap for endoscopic skull base defect repair. Am J Rhinol Allergy. 2009;23(5):522-6.

28. Simal Julian JA, Miranda Lloret P, Cardenas Ruiz-Valdepenas E, et al. Middle turbinate vascularized flap for skull base reconstruction after an expanded endonasal approach. Acta Neurochir (Wien). 2011;153(9):1827-32.

29. Snyderman CH, Janecka IP, Sekhar LN, et al. Anterior cranial base reconstruction: role of galeal and pericranial flaps. Laryngoscope. 1990;100(6):607-14.

30. Georgantopoulou A, Hodgkinson PD, Gerber CJ. Cranial-base surgery: a reconstructive algorithm. Br J Plast Surg. 2003;56(1): 10-3.

31. Patel MR, Shah RN, Snyderman $\mathrm{CH}$, et al. Pericranial flap for endoscopic anterior skull-base reconstruction: clinical outcomes and radioanatomic analysis of preoperative planning. Neurosurgery. 2010;66(3):506-12 discussion 512.

32. Zanation AM, Snyderman CH, Carrau RL, et al. Minimally invasive endoscopic pericranial flap: a new method for endonasal skull base reconstruction. Laryngoscope. 2009;119(1):13-8.

33. Yano T, Tanaka K, Kishimoto S, et al. Reliability of and indications for pericranial flaps in anterior skull base reconstruction. J Craniofac Surg. 2011;22(2):482-5.

34. Fortes FS, Carrau RL, Snyderman CH, et al. Transpterygoid transposition of a temporoparietal fascia flap: a new method for skull base reconstruction after endoscopic expanded endonasal approaches. Laryngoscope. 2007;117(6):970-6.

35. David SK, Cheney ML. An anatomic study of the temporoparietal fascial flap. Arch Otolaryngol Head Neck Surg. 1995;121(10): 1153-6.
36. Rivera-Serrano CM, Oliver C, Prevedello D, et al. Pedicled facial buccinator $(\mathrm{FAB})$ flap: a new flap for reconstruction of skull base defects. Laryngoscope. 2010;120(Suppl 4):S234.

37. Oliver CL, Hackman TG, Carrau RL, et al. Palatal flap modifications allow pedicled reconstruction of the skull base. Laryngoscope. 2008;118(12):2102-6.

38. Hackman T, Chicoine MR, Uppaluri R. Novel application of the palatal island flap for endoscopic skull base reconstruction. Laryngoscope. 2009;119(8):1463-6.

39. Gousheh J, Arab H, Motamed S. Superior cheiloplasty by occipital scalp island flap in male patients. Plast Reconstr Surg. 2003;111(3):1221-6.

40. Rivera-Serrano CM, Snyderman CH, Carrau RL, et al. Transparapharyngeal and transpterygoid transposition of a pedicled occipital galeopericranial flap: a new flap for skull base reconstruction. Laryngoscope. 2011;121(5):914-22.

41. Rivera-Serrano CM, Snyderman CH, Gardner P, et al. Nasoseptal "rescue" flap: a novel modification of the nasoseptal flap technique for pituitary surgery. Laryngoscope. 2011;121(5):990-3.

42. • Kim BY, Shin JH, Kang SG, et al. Bilateral modified nasoseptal "rescue" flaps in the endoscopic endonasal transsphenoidal approach. Laryngoscope. 2013. This is a case series reporting outcomes of another large series of rescue flaps validating the technique and outcomes.

43. - Otto BA, Bowe SN, Carrau RL, et al. Transsphenoidal approach with nasoseptal flap pedicle transposition: Modified rescue flap technique. Laryngoscope. 2013. This is a paper describing a modified technique of a modified nasoseptal rescue flap (NSRF) potentially allowing for greater degree of freedom of the pedicle for clival defects.

44. - Kimple AJ, Leight WD, Wheless SA, et al. Reducing nasal morbidity after skull base reconstruction with the nasoseptal flap: free middle turbinate mucosal grafts. Laryngoscope. 2012; 122(9): 1920-4. This is a small prospective study with retrospective analysis of a larger cohort of patients with the free middle turbinate mucosal graft during use of the nasoseptal flap showing faster septum donor site mucosalization with the grafting technique.

45. Tormenti MJ, Paluzzi A, Pinheiro-Nieto C, et al. Endoscopic endonasal repair of spontaneous CSF fistulae. J Neurosurg. 2012;32(Suppl):E6.

46. Caicedo-Granados E, Carrau R, Snyderman CH, et al. Reverse rotation flap for reconstruction of donor site after vascular pedicled nasoseptal flap in skull base surgery. Laryngoscope. 2010;120(8):1550-2.

47. - Kasemsiri P, Carrau RL, Otto BA, et al. Reconstruction of the pedicled nasoseptal flap donor site with a contralateral reverse rotation flap: Technical modifications and outcomes. Laryngoscope. 2013. This retrospective paper describes outcomes of the first cohort of patients with the reverse rotation flap to re-mucosalize the NSF donor site.

48. Chaaban MR, Chaudhry A, Riley KO, et al. Simultaneous pericranial and nasoseptal flap reconstruction of anterior skull base defects following endoscopic-assisted craniofacial resection. Laryngoscope. 2013. doi:10.1002/lary.24071.

49. Eloy JA, Choudhry OJ, Christiano LD, et al. Double flap technique for reconstruction of anterior skull base defects after craniofacial tumor resection: technical note. Int Forum Allergy Rhinol. 2013;3(5):425-30. 